\title{
Active control of sound radiation due to subsonic wave scattering from discontinuities on fluid-loaded plates. I: Far-field pressure
}

\author{
Y. Gu and C. R. Fuller \\ Department of Mechanical Engineering, Virginia Polytechnic Institute and State University, Blacksburg, \\ Virginia 24061
}

(Received 11 June 1990; revised 1 March 1991; accepted 28 May 1991)

\begin{abstract}
Active control of sound radiation due to subsonic wave scattering from discontinuities represented by a line constraint or by a uniform reinforcing rib positioned on a fluid-loaded infinite plate is analytically studied. The mathematical models are based on the plate vibration and sound radiation due to a line force or a line moment solved in the spectral $k$ domain. For simplicity, the far-field pressure is estimated by the stationary phase approach. Feed-forward control is achieved by adding secondary line forces applied to the plate near the discontinuity. The amplitudes of control forces are determined by the optimal solution of a cost function that integrates the far-field radiated acoustic intensity in a semicylindrical space around the discontinuity. The results show that for subsonic incident waves, high reduction in radiated pressure due to spectral wave scattering at the discontinuities is possible with two active control forces located near the discontinuity. The amount of sound reduction as well as the residual directivity pattern is shown to depend upon the number and location of the control forces.
\end{abstract}

PACS numbers: 43.40.Vn, 43.30.Jx

\section{INTRODUCTION}

Active control of sound radiation has recently attracted substantial attention from acoustical engineers. For well-defined sound radiation patterns, quadratic optimal control theory provides a relatively straightforward mathematical approach to suppress the radiated acoustic power output in a spatially defined acoustic domain. Although the technique of optimizing a quadratic cost function is well known, its application to the control of sound radiation is still a challenging research subject. This approach is well defined by Nelson et al. ${ }^{1}$ and its application to control of noise in cylindrical cavities as well as radiation from panels has been successfully demonstrated. ${ }^{2,3}$

For the past few decades, much work has been carried out analyzing the vibration and sound radiation of fluidloaded plates excited by forces. ${ }^{4-7}$ As well as this, attention has been also directed to the problem of scattering of waves from discontinuities on fluid-loaded plates. ${ }^{8}$ This problem is important because scattering of subsonic, nonradiating waves from discontinuities on fluid-loaded plates can often lead to radiation of acoustic power. ${ }^{4}$ As discontinuities are present on all physical systems such as marine vessels and aircraft, this phenomenon is important in terms of noise control.

The paper is concerned with applying active control to reducing the sound radiation due to wave scattering from discontinuities. The active control is achieved by applying forces near the discontinuities and the control approach is a steady state feed-forward model. The optimal control forces are obtained using quadratic minimization of a cost function based on the radiated acoustic power. For this initial investigation, the analysis is based on an idealized system consisting of infinite thin plate with semi-infinite acoustic fluid on one side and in vacuo on the other. The discontinuities con- sidered consist of a line constraint or a uniform rib attached to the plate. The mathematical approach is to describe the response of the plate system in the spectral $k$ domain and solve for the far-field radiated pressure using the stationary phase approach. For the numerical examples presented here the acoustic field is considered to be water ensuring strong coupling between plate and fluid response. The incident acoustic waves are limited to subsonic, thus all noise radiation to the far field is due to wave scattering phenomena.

Although the infinite plate system is idealized, the paper does consider two new themes: active control of sound radiation from fluid-loaded coupled structures as well as control of radiation due to line discontinuities. The results thus add new understanding to this important problem.

\section{ANALYSIS}

The infinite plate is considered to be loaded with an acoustic fluid on one side and in vacuo on the other. There are two forms of discontinuities to be studied. The first discontinuity is of a line constraint at which plate displacement is constrained to zero. When the subsonic incident flexural wave impinges on the constraint it interacts with the constraint causing the discontinuity to exert a reaction line force on the plate. This line force generates additional plate waves and associated radiation, which represents the scattered fields. The second discontinuity consists of a reinforcing rib attached to the plate. In this case the rib is assumed to act as a lumped mass that has both translational and rotational inertia and thus can be approximated as exerting both a reaction moment as well as a force on the plate. In this case these reaction inputs will be related to the inertial properties of the rib as driven by the incident field on the plate. Both of these cases are shown in Fig. 1.

Thus, before considering control of sound radiation 

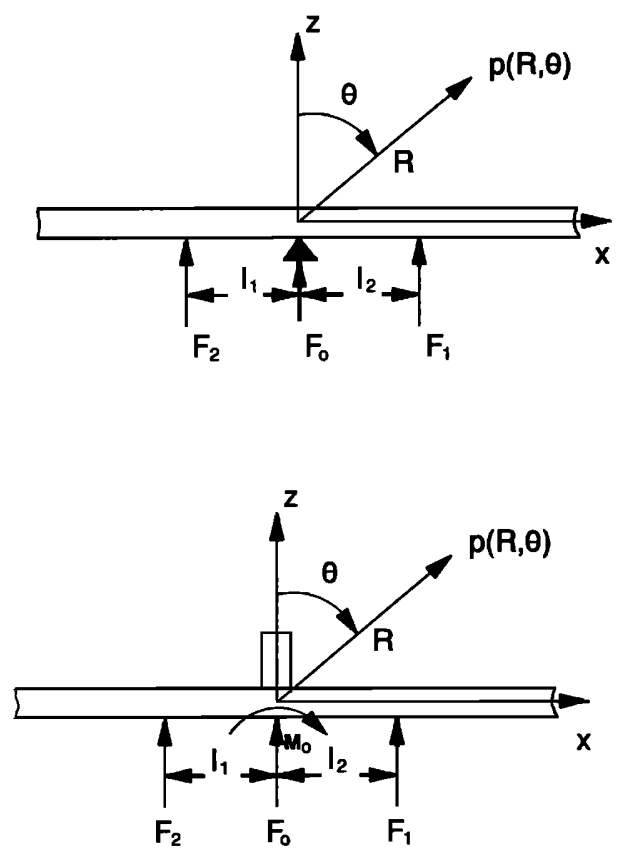

FIG. 1. (a) Arrangement of line constraint discontinuity. (b) Arrangement of rib constraint discontinuity.

from these discontinuities, it is appropriate to review radiation from plates driven by line forces and moments.

\section{A. Radiation due to a line force}

Consider the infinite plate as shown in Fig. 1(a) which is excited by a harmonic line force at $x=0$,

$$
F(x, t)=F_{0} \delta(x) e^{-i \omega t},
$$

where $\omega$ is the frequency of excitation, $F_{0}$ is the complex magnitude, and $\delta(\cdot)$ is the Dirac delta function.

With a $k$-plane Fourier transform and associated manipulations, the normalized plate out-of-plane velocity can be expressed as the sum of residue terms and two branch integrals. ${ }^{4}$ These residues and branch cut integrals can be evaluated using the procedure developed by Nayak. ${ }^{4}$ Thus the response of the plate is evaluated as

$$
\begin{aligned}
\bar{v}_{F}(X) & =\frac{v(X)}{k_{p} F_{0} / 4 \omega m} \\
& =-\frac{2 i}{\pi} \int_{-\infty}^{\infty} \frac{\left(\alpha^{2}-\bar{k}_{x}^{2}\right)^{1 / 2} e^{i \bar{k}_{x} X}}{\left(\bar{k}_{x}^{4}-1\right)\left(\alpha^{2}-\bar{k}_{x}^{2}\right)^{1 / 2}-i \xi / \alpha} d \bar{k}_{x},
\end{aligned}
$$

where $k_{p}=\left(\omega^{2} m / D\right)^{1 / 4}$ is the in-vacuo flexural wavenumber, $m$ is the mass per unit area, $D=E h^{3}\left[12\left(1-v^{2}\right)\right]$ is the bending rigidity, $E$ is the Young's modulus, $v$ is the Poisson's ratio, and $h$ the thickness of the plate, respectively. Other important terms are $\alpha=k_{0} / k_{p}, k_{0}=\omega / c_{0}$ the acoustic wave number in the fluid, $\bar{k}_{x}=k_{x} / k_{p}$ the nondimensional wave number, $X=k_{p} x$ the nondimensional distance, $\xi=\rho_{0} c_{0} / \omega_{c} m$ the fluid loading parameter, in which $\rho_{0}$ is the acoustic fluid density, and $c_{0}$ the sound speed in the fluid, and $\omega_{c}=c_{0}^{2}(m / D)^{1 / 2}$ the coincidence frequency. The meanings of $\xi$ and $\omega_{c}$ are well defined in Refs. 5-7.

The integral of Eq. (2) can be solved using contour integration as outlined by Nayak. ${ }^{4}$ Before carrying this out it is necessary to find the system poles. The location of the system poles can be obtained by solving the system characteristic equation that has been extensively studied by Crighton ${ }^{7}$ and Junger and Feit. ${ }^{9}$ The system characteristic equation is

$$
\left(\bar{k}_{x}^{4}-1\right)\left(\alpha^{2}-\bar{k}_{x}^{2}\right)^{1 / 2}-i \xi / \alpha=0 .
$$

If $\bar{k}_{z}=\left(\alpha^{2}-\bar{k}_{x}\right)^{1 / 2}$, the roots of the characteristic equation should be chosen such that $\operatorname{Im}\left(\bar{k}_{z}\right) \geqslant 0$ or when $\operatorname{Im}\left(\bar{k}_{z}\right)=0, \operatorname{Re}\left(\bar{k}_{z}\right) \geqslant 0$ in order to satisfy the Sommerfield radiation condition ${ }^{4}$ (see Fig. 2). The meanings of poles in Fig. 2 were well explained by previous researchers. ${ }^{4,7}$

The associated acoustic field pressure in the top halfplane is given by ${ }^{6}$

$$
\begin{aligned}
\bar{p}_{F}(X, Z) & =\frac{p_{F}(X, Z)}{F_{0} k_{0} \rho_{0} c_{0} / 4 \omega m} \\
& =-\frac{2 i}{\pi} \int_{-\infty}^{\infty} \frac{\exp \left[i\left(\bar{k}_{x} X+\sqrt{\alpha^{2}-\bar{k}_{x}^{2}} Z\right)\right]}{\left(\bar{k}_{x}^{4}-1\right)\left(\alpha^{2}-\bar{k}_{x}^{2}\right)^{1 / 2}-i \xi / \alpha} d \bar{k}_{x},
\end{aligned}
$$

where $\bar{p}_{F}$ is the nondimensional pressure caused by a line force $F_{0}$.

For the far-field pressure, Eq. (4) is evaluated using the stationary phase approach as

$$
\bar{p}_{F}(R, \theta)=\frac{2 \mu \cos \theta e^{i\left(k_{0} R-\pi / 4\right)}}{1+i \mu\left(\alpha^{4} \sin ^{4} \theta-1\right) \cos \theta},
$$

where $\bar{p}_{F}(R, \theta)=p_{F}(R, \theta) / c_{F}$ and $c_{F}=\rho_{0} F_{0} / 2 \sqrt{2 \pi R k_{0}} m$. Throughout the paper, the plate out-of-plane velocity, the near-field pressure, and the far-field pressure are evaluated using Eqs. (2), (4), and (5), respectively.

\section{B. Radiation from a line moment}

If the plate is restrained by a rib instead of being supported by a line constraint, there will be both a line force and a line moment generated by incident wave impinging on the rib.

The radiation due to a line force has already been derived, and a similar approach will be taken for the line mo-

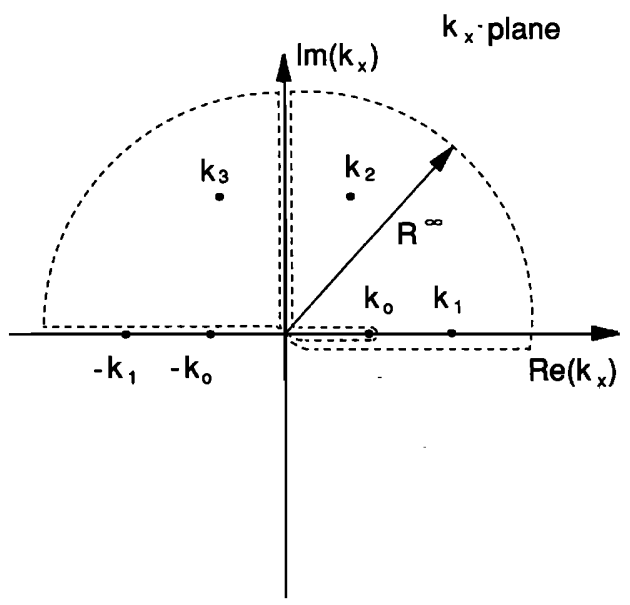

FIG. 2. Integration contour in $k$ plane and locations of roots. 
ment excitation. Using a $k$-Fourier transform approach the response of a plate due to a line moment is

$$
M(x, t)=M_{0} \delta^{\prime}(x) e^{-i \omega t},
$$

where $M_{0}$ is the complex magnitude and $\delta^{\prime}(\cdot)$, and the derivative of the Delta function with respect to its argument, can be written as $\delta^{\prime}(x)=(d / d x)[\delta(x)]$.

The associated acoustic field has been derived by Liu and Rumerman $^{5}$ as

$$
\begin{aligned}
\bar{p}_{M}(X, Z)= & \frac{p_{M}(X, Z)}{M_{0} k_{p} k_{0} \rho_{0} c_{0} / 4 \omega m} \\
= & -\frac{2 i}{\pi} \\
& \times \int_{-\infty}^{\infty} \frac{i \bar{k}_{x} \exp \left[i\left(\bar{k}_{x} X+\sqrt{\alpha^{2}-\bar{k}_{x}^{2}} Z\right)\right]}{\left(\bar{k}_{x}^{4}-1\right)\left(\alpha^{2}-\bar{k}_{x}^{2}\right)^{1 / 2}-i \xi / \alpha} d \bar{k}_{x},
\end{aligned}
$$

where $\bar{p}_{M}$ is the nondimensional pressure caused by a line moment $M_{0}$.

Similar to the derivation of Eq. (5), the far-field radiated pressure due to a line moment is derived with stationary phase approach as

$$
\bar{p}_{M}(R, \theta)=\frac{-2 i \mu \sin \theta \cos \theta e^{i\left(k_{u} R-\pi / 4\right)}}{1+i \mu\left(\alpha^{4} \sin ^{4} \theta-1\right) \cos \theta},
$$

where $\bar{p}_{M}(R, \theta)=p_{M}(R, \theta) / c_{M}$ and $c_{M}=\rho_{0} M_{0} k_{p}^{1 / 2} /$ $2 \sqrt{2 \pi R} m$.

\section{Radiated pressure field from line constraint with control forces}

The configuration of the line constraint system is shown in Fig. 1 (a). When an incident flexural wave impinges on the discontinuity, the line constraint effectively exerts a back reaction force $\widehat{F}_{0}$ on the plate where the caret denotes the complex amplitude of the force. The other two forces $\widehat{F}_{1}$ and $\widehat{F}_{2}$ represent control forces applied to the plate. For a flexural wave input (the noise field) of

$$
w_{i}(x, t)=\hat{W}_{0} e^{i k_{i} X-i \omega t},
$$

where $w_{i}$ is the incident wave displacement and $k_{i}$ is the incident wave number, the flexural wave velocity will equal $v_{i}(x, t)=\widehat{V}_{0} e^{i k_{i} X-i \omega t}$, where $\hat{V}_{0}=-i \omega \hat{W}_{0}$ and the wave rotation displacement is

$$
\frac{\partial v_{i}(x, t)}{\partial x}=i k_{i} k_{p} \hat{V}_{0} e^{i k_{r} X-i \omega t}=i k_{i} k_{p} v_{i}(x, t) .
$$

By superposition the total velocity response of the plate system to the back reaction force and the control forces can be written, at $x=0$, as

$v(0, t)=\left(\widehat{V}_{0}+\frac{\widehat{F}_{0} k_{p}}{4 \omega m} \bar{v}(0)+\sum_{n=1}^{N} \frac{\hat{F}_{n} k_{p}}{4 \omega m} \bar{v}\left(k_{p} l_{n}\right)\right) e^{-i \omega t}$,

where $n$ is the number of control forces and $\bar{v}(x)$ is given by Eq. (2). Applying the boundary condition of the line constraint that out-of-plane displacement is zero at $x=0$ gives

$$
\widehat{F}_{0}=\sum_{n=1}^{N} \widehat{F}_{n} v_{n}+v^{\prime}
$$

where $v_{n}=-\bar{v}\left(k_{p} l_{n}\right) / \bar{v}(0)$, and $v^{\prime}=-4 \omega m \widehat{V}_{0} / k_{p} \bar{v}(0)$, and the time variation is omitted.

Since the control forces are located near the line constraint, the distance to the observation point from all forces is almost identical, thus only the phase shift is considered in the pressure radiated by the control forces while the nondimensional magnitudes of the pressure radiation due to the control forces are considered the same as that of the constraint reaction force. The pressure field radiated from the control forces can thus be approximately expressed as

$$
\bar{p}_{n}(R, \theta) \simeq \bar{p}_{F}(R, \theta) \exp \left[-i \operatorname{sgn}\left(l_{n}\right) k_{0} l_{n} \sin \theta\right]
$$

for a control force $n$, positioned at $x=l_{n}$, and $\bar{p}_{F}(R, \theta)$ is given by Eq. (4). Note that the approximation holds when $\left|l_{n}\right| \ll R$ and "sgn" is a sign function that either takes +1 or -1 according to positive or negative values of $l_{n}$.

Using Eq. (11) as the solution for the back reaction force, the total plate radiated pressure can be expressed in terms of the incident wave amplitude (usually assumed known) and the unknown control force amplitudes, $\widehat{F}_{n}$, as

$$
p_{\text {total }}^{\text {rad }}(R, \theta)=b_{1}+\sum_{n=1}^{N} a_{n} \widehat{F}_{n},
$$

where

$$
\begin{aligned}
a_{n}= & \left(\rho_{0} / 2 \sqrt{2 \pi R k_{0}} m\right) \bar{p}_{F}(R, \theta) \\
& \times\left\{\exp \left[-i \operatorname{sgn}\left(l_{n}\right) k_{0} l_{n} \sin \theta\right]+v_{n}\right\}
\end{aligned}
$$

and

$$
b_{1}=\left(\rho_{0} / 2 \sqrt{2 \pi R k_{0}} m\right) \bar{p}_{F}(R, \theta) v^{\prime},
$$

or be written in vector form as

$$
p_{\text {total }}^{\mathrm{rad}}(R, \theta)=a^{T} \hat{F}+b,
$$

where $a^{T}=\left[a_{1}, a_{2}, \cdots, a_{n}\right]$ and $b=b_{1}$.

\section{Radiated pressure from rib constraint with control forces}

For the rib-plate system the derivation is very similar, but the form of the boundary condition is different. In this case it is assumed that the rib stays attached to the plate, thus the rib out-of-plate displacement and rotation (taken as a lumped parameter system) must equal the plate at the point of contact, $x=0$. Thus when an incident flexural wave strikes the rib, it exerts both an inertial back reaction force and moment as depicted in Fig. 1(b). Note that the actual total displacements and rotations are not constrained to some fixed values as in the line constraint case. The application of control forces will lead to markedly different behavior.

Besides Eq. (2), the translational and rotational plate velocities due to a line force or moment can all be obtained by following Nayak's approach. ${ }^{4}$ These admittances were given by Liu and Rumerman ${ }^{5}$ as follows: 


$$
\begin{aligned}
\bar{\theta}_{F}(X)= & \frac{\partial v(X) / \partial x}{F_{0} k_{p}^{2} / 4 \omega m} \\
= & -\frac{2 i}{\pi} \int_{-\infty}^{\infty} i \bar{k}_{x} \\
& \times \frac{\left(\alpha^{2}-\bar{k}_{x}^{2}\right)^{1 / 2} e^{i \bar{k}_{x} X}}{\left(\bar{k}_{x}^{4}-1\right)\left(\alpha^{2}-\bar{k}_{x}^{2}\right)^{1 / 2}-i \xi / \alpha} d \bar{k}_{x}, \\
\bar{v}_{M}(X)= & \frac{v(X)}{M_{0} k_{p}^{2} / 4 \omega m}=-\bar{\theta}_{F}(X), \\
\bar{\theta}_{M}(X)= & \frac{\partial v(X) / \partial x}{M_{0} k_{p}^{3} / 4 \omega m} \\
= & -\frac{2 i}{\pi} \int_{-\infty}^{\infty} \bar{k}_{x}^{2} \\
& \times \frac{\left(\alpha^{2}-\bar{k}_{x}^{2}\right)^{1 / 2} e^{i \bar{k}_{x} X}}{\left(\bar{k}_{x}^{4}-1\right)\left(\alpha^{2}-\bar{k}_{x}^{2}\right)^{1 / 2}-i \xi / \alpha} d \bar{k}_{x} .
\end{aligned}
$$

Equations (2), (17), (18), and (19) indicate the translational velocity due to line force, the rotational velocity due to a line force, the translational velocity due to a line moment, and the rotational velocity due to a line moment, respectively, where the line force or moment is located at $X=0$. The procedure to evaluate these admittances will follow the same Cauch residue approach used in evaluating Eq. (2). For brevity these numerical calculation procedures are omitted here since they are very similar to the approach introduced in Sec. I A.

Following the procedure of the previous section, the total velocity responses of the plate-rib system with an incident wave and control forces can be written as

$$
\begin{aligned}
v(0, t)= & \left(\widehat{V}_{0}+\frac{\widehat{F}_{0} k_{p}}{4 \omega m} \bar{v}_{F}(0)+\frac{\widehat{M}_{0} k_{p}^{2}}{4 \omega m} \bar{v}_{M}(0)\right. \\
& \left.+\sum_{n=1}^{N} \frac{\widehat{F}_{n} k_{p}}{4 \omega m} \bar{v}_{F}\left(k_{p} l_{n}\right)\right) e^{-i \omega t}
\end{aligned}
$$

and

$$
\begin{aligned}
\dot{\theta}(0, t)= & \left(\frac{\partial \hat{V}_{0}}{\partial x}+\frac{\widehat{F}_{0} k_{p}^{2}}{4 \omega m} \bar{\theta}_{F}(0)+\frac{\widehat{M}_{0} k_{p}^{3}}{4 \omega m} \bar{\theta}_{M}(0)\right. \\
& \left.+\sum_{n=1}^{N} \operatorname{sgn}\left(l_{n}\right) \frac{\widehat{F}_{n} k_{p}^{2}}{4 \omega m} \bar{\theta}_{F}\left(\left|k_{p} l_{n}\right|\right)\right) e^{-i \omega t} .
\end{aligned}
$$

With the approach used by Junger and Feit ${ }^{9}$ and Cremer and Heckl $^{10}$ of dealing with the scattering of flexural wave by a plate discontinuity and some manipulations, the back reaction force and moment

$$
\widehat{F}_{0}=\sum_{n=1}^{N} \widehat{F}_{n} v_{n}^{\prime}+v^{\prime \prime},
$$

where

$$
v_{n}^{\prime}=\left(\frac{i M_{r} k_{p} \bar{v}_{F}(0)}{4 m-i M_{r} k_{p} \bar{v}_{F}(0)}\right) \frac{\bar{v}_{F}\left(k_{p} l_{n}\right)}{\bar{v}_{F}(0)}
$$

and

$$
v^{\prime \prime}=\left(\frac{i M_{r} k_{p} \bar{v}_{F}(0)}{4 m-i M_{r} k_{p} \bar{v}_{F}(0)}\right) \frac{4 \omega m \widehat{V}_{0}}{k_{p} \bar{v}_{F}(0)},
$$

in which $M_{r}$ and $M_{r}$ are the mass and rotational inertia of the rib per unit length, respectively, and

$$
\widehat{M}_{0}=\sum_{n=1}^{N} \widehat{F}_{n} u_{n}+u^{\prime}
$$

where

$$
u_{n}=\left(\frac{i J_{r} k_{p}^{3} \bar{\theta}_{M}(0)}{4 m-i J_{r} k_{p}^{3} \bar{\theta}_{M}(0)}\right) \operatorname{sgn}\left(l_{n}\right) \frac{\bar{\theta}_{F}\left(\left|k_{p} l_{n}\right|\right)}{k_{p} \bar{\theta}_{M}(0)}
$$

and

$$
u^{\prime}=\left(\frac{i J_{r} k_{p}^{3} \bar{\theta}_{M}(0)}{4 m-i J_{r} k_{p}^{3} \bar{\theta}_{M}(0)}\right) \frac{4 \omega m}{k_{p}^{3} \bar{\theta}_{M}(0)} \frac{\partial \widehat{V}_{0}}{\partial x} .
$$

The total radiated pressure field due to the rib back reaction force and control inputs can be expressed in terms of control forces as

$$
p_{\text {total }}^{\mathrm{rad}}(R, \theta)=b_{1}+\sum_{n=1}^{N} a_{n} \widehat{F}_{n},
$$

where

$$
\begin{aligned}
a_{n}= & \left(\rho_{0} / 2 \sqrt{2 \pi R k_{0}} m\right)\left\{\bar{p}_{F}(R, \theta)\right. \\
& \times \exp \left[-i \operatorname{sgn}\left(l_{n}\right) k_{0} l_{n} \sin \theta\right]+\bar{p}_{F}(R, \theta) v_{n}^{\prime} \\
& \left.+k_{p} \bar{p}_{M}(R, \theta) u_{n}\right\},
\end{aligned}
$$

and

$$
b_{1}=\left(\rho_{0} / 2 \sqrt{2 \pi R k_{0}} m\right)\left[\bar{p}_{F}(R, \theta) v^{\prime \prime}+k_{p} \bar{p}_{M}(R, \theta) u^{\prime}\right] .
$$

Since the form of Eq. (28) is the same as Eq. (13), it can also be rewritten in vector form analogous to Eq. (16).

\section{E. Optimal solution for feed-forward control}

The optimization technique for feed-forward control relies on forming a quadratic function in the control force amplitudes and finding the minimum of that function; the optimal solution. Usually this is achieved by squaring an appropriate response variable of the system. ${ }^{1,3}$

Thus, in order to derive the optimal amplitude of the control forces so as to reduce the radiated noise, we need to define a suitable cost function. A suitable cost function in this case is the total radiated power from the plate to the halfspace far field. Thus the cost function is given by

$$
\beta\left(\widehat{F}_{n}\right)=\int_{s}\left|p_{\text {total }}^{\text {rad }}\right|^{2} d s,
$$

where $s$ is the semicylindrical half-space surface in the far field at some arbitrary distance $R$.

Substituting the expressions for total radiated pressure for the line constraint or the rib constraint, Eq. (16) or (28) into Eq. (31), respectively, the cost function becomes

$$
\beta\left(\widehat{F}_{n}\right)=F^{T} A F^{*}+F^{T} B+B^{H} F^{*}+C,
$$

where $\quad A=\int_{s}\left[a a^{H}\right] d s, \quad B=\int_{s}\left[b a^{H}\right] d s, \quad$ and $\quad C$ $=\int_{s}\left[b b^{H}\right] d s$.

Note that in expressions $A, B$, and $C, a$ and $b$ have different definitions when line constraint or rib constraint is considered and in the above equations " $H$ " denotes the transpose conjugate operator. The cost function is a real scalar function of the complex control vector $F$. Here, $A$ is a Hermi- 
tian matrix, i.e., $A^{T}=A^{*}$. When two control forces are used, and are symmetrically located, $A$ becomes a real symmetric matrix.

In order to derive the optimal control forces, the cost function is differentiated with respect to the control forces and set to zero, as outlined in Refs. 1 and 2. The optimal solution of Eq. (32) becomes

$$
F_{\mathrm{opt}}=-A^{*-1} B^{*} \text {, }
$$

where the optimal control force vector is given by, for $N$ forces,

$$
F_{\text {opt }}^{T}=\left[\hat{F}_{1} \hat{F}_{2} \cdots \hat{F}_{N}\right] .
$$

\section{RESULTS AND DISCUSSION}

For the system studied, the plate is assumed infinite, thin, elastic, and immersed in a fluid on one side. The media chosen were steel and water with material properties dimensions given in Table I. The incident flexural wave was assumed to have a displacement amplitude $\hat{W}_{0}=1+0 i \mathrm{~mm}$. Two subsonic frequencies, 1170.8 and $11708 \mathrm{rad} / \mathrm{s}$, were considered as example calculations. These frequencies were equivalent to values of $\omega / \omega_{c}$ equal to 0.02 and 0.2 , where the critical frequency for this system is $\omega_{c}=58538 \mathrm{rad} / \mathrm{s}^{6,9}$ In all the directivity patterns it was assumed that the observation point was at $R=10 \mathrm{~m}$ centered on the line constraint.

For the rib constraint, the rib was assumed to be made of steel with dimensions and properties also given in Table I. For the results presented here, either one or two control forces were considered as control inputs. When one control force is used, the matrices in the optimal analysis degenerate to scalars, however the approach remains the same.

The first step in the calculation of radiated pressure with and without control is to evaluate the $A$ and $B$ matrices in the cost functions. The approach used here follows closely that of Feit and Liu, ${ }^{6}$ in which the residue contributions are evaluated explicitly (this also involves solving the system characteristic equation of which the poles are discussed by Crighton $^{7}$ and Junger and Feit ${ }^{9}$ in detail ) and the branch cut contributions were evaluated numerically using a GaussKronrod integral approach. This procedure can be quite difficult and the authors refer the reader to the excellent work by Feit and Liu $^{6}$ that discusses the major pitfalls. The work of Feit and $\mathrm{Liu}^{6}$ also studies the response of an identical system as considered here to a line force and details the radiation patterns of the far field and near field under the same frequencies. These will be seen to correspond to the noise or primary field considered here.
The results are plotted in terms of the far-field radiation directivity pattern centered on the discontinuity. For convenience in the radiation plots, negative values of radiation angle $\theta$ correspond to negative axial coordinate positions in $\boldsymbol{x}$.

\section{A. Line constraint, $\omega / \omega_{c}=0.02$}

Figure 3 presents the sound pressure directivity patterns in the far field for $\omega / \omega_{c}=0.02$. It is apparent that in Fig. 3 the noise field is monopole-like, being fairly uniform with radiation angle. This is to be expected since the line constraint acts as a single back-reaction force.

When one control force is applied at $l_{1}=0.36 \mathrm{~m}$ on the right side of the constraint, the radiated pressure is markedly reduced ly around 15 to $25 \mathrm{~dB}$. The residual sound field has the shape of a dipole radiation source, due to the control force combining with the back reaction force to create a moment-like input to the plate (as they are not colocated). Note that applying the control force at $x=0$ will lead to no reduction, since due to the boundary condition, the input impedance is infinite at this point. Studies have also shown that either increasing or decreasing the control location will lead to a deterioration in amount of reduction. There is an optimal location for the control force location and this will be further studied in a companion paper.

When two control forces at $l_{1}=0.16 \mathrm{~m}$ and $l_{2}=-0.16$ $\mathrm{m}$ are applied, there is a further increase in the amount of sound reduction. The residual radiation field is now symmetric and has the radiation pattern associated with a high order source.

It is interesting to note that there are two alternate ways to view the control action. On one hand from the "superposition" point of view the control action is effectively reducing the back reaction force from the discontinuity thus reducing the monopole radiation term. Another point of view is to consider that the reaction force in concert with the control force creates a radiation source that is higher order with an associated lower radiation efficiency with little significant change in spatially averaged plate response amplitudes.

\section{B. Line constraint, $\omega / \omega_{c}=0.2$}

For the next two cases the input frequency was increased. Figure 4 shows that at this higher frequency the radiation field is still monopole-like but has significantly increased in amplitude due to plate mobility increasing with frequency.

When one control force is employed, located at $x$ $=0.12 \mathrm{~m}$, there is sound reduction but not nearly to the

TABLE I. Material properties.

\begin{tabular}{lllll}
\hline \hline System & $\begin{array}{l}\text { Phase } \\
\text { speed }(\mathrm{m} / \mathrm{s})\end{array}$ & $\begin{array}{l}\text { Density } \\
\left(\mathrm{kg} / \mathrm{m}^{3}\right)\end{array}$ & $\begin{array}{l}\text { Thickness } \\
(\mathrm{m})\end{array}$ & $\begin{array}{l}\text { Incident wave } \\
\text { magnitude (mm) }\end{array}$ \\
\hline $\begin{array}{l}\text { Steel plate } \\
\text { Water }\end{array}$ & 2916 (shear) & 7700 & 0.0254 & $1+0 i$ \\
& 1500 & 1026 & & Width (m) \\
Rib & & $\begin{array}{l}\text { Density }\left(\mathrm{kg} / \mathrm{m}^{3}\right) \\
0.05\end{array}$ & 0.127 \\
\hline \hline
\end{tabular}




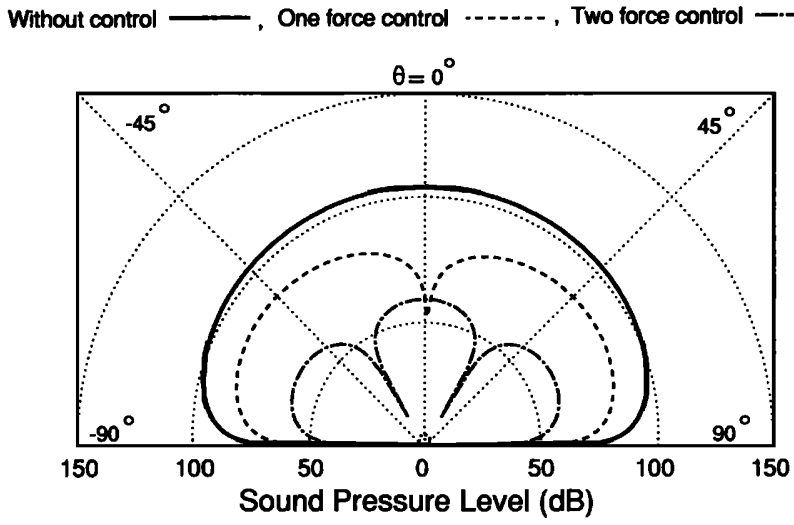

FIG. 3. Directivity pattern of radiation, the line constraint case: $\omega / \omega_{c}=0.02$.

degree of the lower frequency as shown in Fig. 3. In this case, the control force location was again varied to find an optimal location. Note that its axial position is less than the lower frequency most likely due to the shorter wavelengths for this case. These effects will be discussed in more detail in the companion paper which focuses on plate responses. ${ }^{\text {1 }}$

When the number of control forces is increased to two ( $l_{1}=0.02 \mathrm{~m}, l_{2}=-0.02 \mathrm{~m}$ ) improved control is achieved, with reductions of 25 to $35 \mathrm{~dB}$ being observed. It is apparent that as the frequency of excitation is increased the importance of using an additional second control force also increases.

\section{Rib constraint, $\omega / \omega_{c}=0.02$}

The sound radiation from a rib constraint is more complicated since the noise source consists of a line force as well as a line moment. The combination of such sources will increase the difficulty of control, as we will see in the following results.

Figure 5 gives the radiation directivity patterns for an excitation frequency of $\omega / \omega_{c}=0.02$. In this case control is applied by a single force located at $l=0.0054 \mathrm{~m}$. It is apparent from Fig. 5 that the noise field is still monopole-like for the rib system. Although the rib exerts both a back reaction

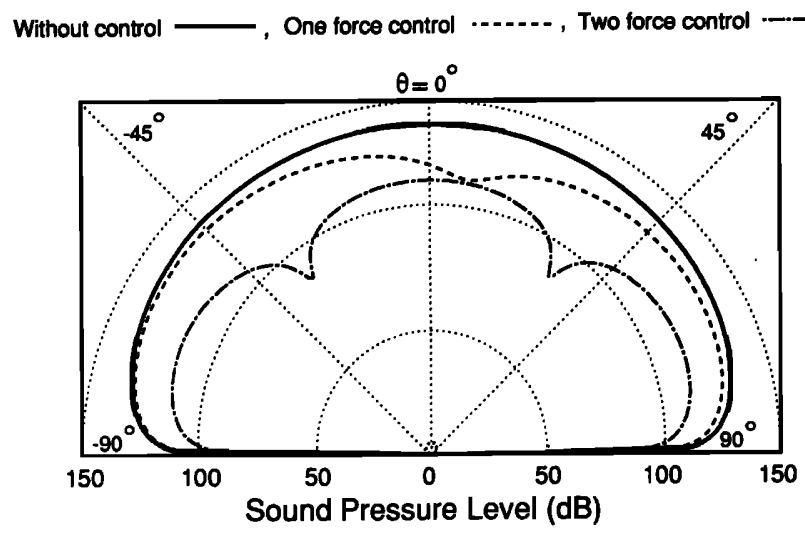

FIG. 4. Directivity pattern of radiation, the line constraint case: $\omega / \omega_{c}=0.2$.

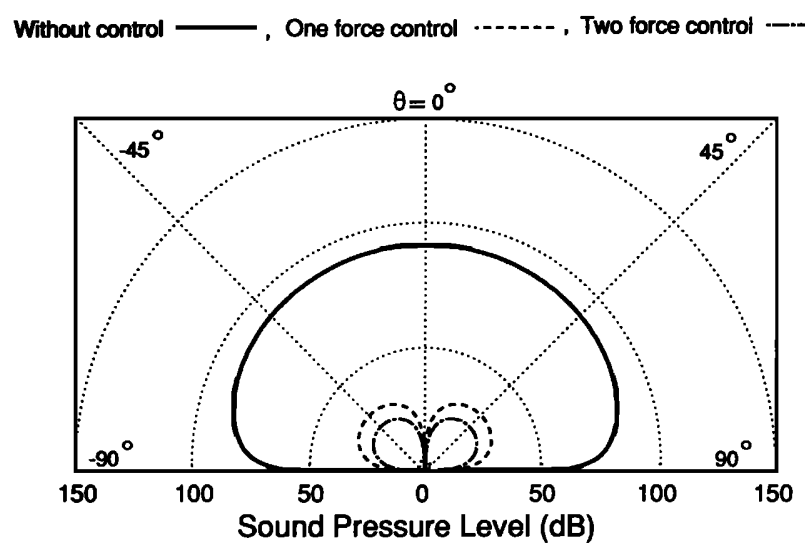

FIG. 5. Directivity pattern of radiation, the rib constraint case: $\omega / \omega_{c}=0.02$.

moment as well as a force, the latter is a far more efficient source and thus its contribution dominates the radiation field.

When control is applied the sound field is markedly reduced by 60 to $80 \mathrm{~dB}$. Note that the directivity pattern is again dipole-like since the main action of the control force is to cancel out the rib inertia force $F_{0}$ leaving the dipole like radiation from the dipole term. It is also interesting to note that when the control force is located at $x=0$ the amount of reduction is not as great. Thus offsetting the force allows control of the back reaction force as well as creating a control moment input, thus simultaneously controlling both source terms.

When two control forces are symmetrically applied at $l_{1}=0.0013 \mathrm{~m}$ and $l_{2}=-0.0013 \mathrm{~m}$, a further sound reduction can be seen in Fig. 5. The results also reveal that the closer the two control forces are, the larger the reduction can be achieved. This is reasonable since the two control forces are able to cancel the sound radiation from a line force as well as from a line moment. A very short distance between the two control forces will better simulate a control line moment which will lead to improved suppression of the moment radiation due to the rib rotational inertia. In practice it will be difficult to implement two extremely closely spaced forces and a design compromise will be necessary.

\section{Rib constraint, $\omega / \omega_{c}=0.2$}

For an increased input frequency, a similar result as the previous case is found with one control force located at $l_{1}=0.0092 \mathrm{~m}$ as shown in Fig. 6 . However in this case the amount of reduction achieved is reduced to around 30 to 50 $\mathrm{dB}$. This result tends to indicate that the radiation term from the moment reaction becomes more important as the rate of rotational acceleration of the rib increases with $\omega^{2}$, and plate wavelengths become shorter.

With two control forces symmetrically located at $l_{1}=0.0001 \mathrm{~m}$ and $l_{2}=-0.0001 \mathrm{~m}$, increased noise reduction is achieved. However Fig. 6 illustrates some interesting aspects. Although two control forces were used it was not possible to totally attenuate the radiated sound. This result is most likely due to the fact that the rib theoretically exerts its 


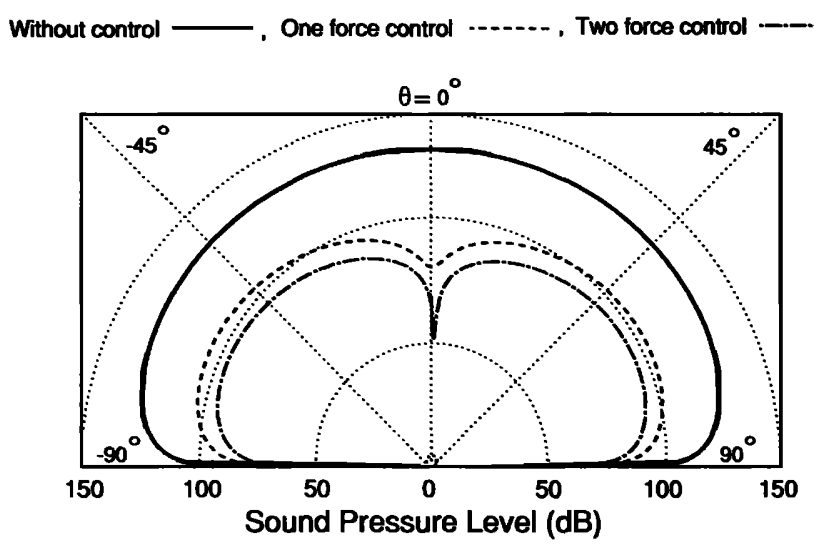

FIG. 6. Directivity pattern of radiation, the rib constraint case: $\omega / \omega_{c}=0.2$.

reaction moment at a line while the control moment input is distributed over $x$. Thus the control input cannot completely "cancel" the rib moment unless the two control forces can be implemented on a single line, i.e., as a pure line moment.

\section{CONCLUDING DISCUSSION}

Active control of sound radiation from a fluid-loaded plate with subsonic waves incident upon either a line constraint or an uniform rib has been analytically studied. The control approach is based upon quadratic optimization of the total acoustic power radiated into the fluid half space.

The results demonstrate that for both discontinuity cases, significant reductions in the radiated sound pressures can be achieved with two control forces located near the discontinuity. The efficiency of sound reduction was demonstrated to be strongly dependent upon frequency and the location of the control forces. In general, as the frequency is increased the role of the second control force increases in importance.

The amount of reduction obtainable was also shown to be influenced by the nature of the discontinuity. In general, better control was achieved for fixed boundary conditions such as the line constraint than for free boundary conditions such as the rib.

This study adds new understanding to research in scattering from fluid-loaded discontinuities. The results indicate that sound radiation from subsonic waves impinging upon discontinuities can be suppressed by active structural inputs near the discontinuity. The approach is also extendable to other plate boundary conditions and finite plates. The plate vibrational response and intensity distributions will be extensively studied in accompanying papers.

\section{ACKNOWLEDGMENTS}

The authors gratefully acknowledge the support of this work by the Office of Naval Research under Grant ONRN00014-88-K-0721 and are also grateful to Dr. Y. N. Liu of DTNSRC for many helpful discussions on aspects of the behavior of fluid-loaded plates.

'P. A. Nelson, A. R. D. Curtis, S. J. Elliott, and A. J. Bullmore, "The minimum power output of free field point sources and the active control of sound," J. Sound Vib. 116, 397-414 (1987).

${ }^{2}$ H. C. Lester and C. R. Fuller, “Active control of propeller induced noise fields inside a flexible cylinder," AIAA Paper 86-1957 (1986).

${ }^{3}$ C. R. Fuller, "Analysis of active control of sound radiation from elastic plates by force inputs," Proceedings of Inter Noise '88, Avignon, France, 1061-1064 (1988).

${ }^{4}$ R. P. Nayak, "Line admittance of infinite isotropic fluid-loaded plates," J. Acoust. Soc. Am. 47, 191-201 (1971).

${ }^{5}$ Y. N. Liu and M. L. Rumerman, "The Effects of Adjacent Elastic Structures on Radiation by Acoustic Volume Sources," David W. Taylor Naval Ship Research and Development Center Report, SAD-335E-1965, pp. $1-20$ (1981).

${ }^{6} \mathrm{D}$. Feit and Y. N. Liu, "The near-field response of a line-driven fluidloaded plate," J. Acoust. Soc. Am. 78, 763-766 (1985).

${ }^{7}$ D. G. Crighton, "The free and forced waves on a fluid loaded elastic plate," J. Sound Vib. 63, 225-235 (1979).

${ }^{8}$ M. S. Howe and M. Heckl, "Sound radiation from plates with density and stiffness discontinuities," J. Sound Vib. 21, 193-203 (1972).

${ }^{9}$ M. C. Junger and D. Feit, Sound, Structures, and Their Interaction (MIT Press, Cambridge, MA, 1986), pp. 235-257.

${ }^{10} \mathrm{~L}$. Cremer and M. Heckl, Structure-Borne Sound (Springer-Verlag, Berlin, 1988), pp. 385-389.

"Y. Gu and C. R. Fuller, "Active control of sound radiation due to subsonic wave scattering from discontinuities on fluid-loaded plates. II: Plate response," to be submitted to J. Acoust. Soc. Am. (1991). 